\title{
Monte Carlo developments and data comparison
}

\author{
Rikkert Frederix* \\ Physik Department T31, Technische Universität München, James-Franck-Str. 1, \\ D-85748 Garching, Germany \\ E-mail: rikkert.frederixatum.de
}

During the last years, the improvement in predictions for LHC processes has been enormous. In this talk the following two topics are covered. First, using the FxFx merging prescription, the prediction and data comparison for a vector boson in association with multiple jets are considered at NLO accuracy. Second, we show some results for the newly developed extension of the MINLO method that allows, by way of imposing numerical unitarity, the elimination of a merging scale for arbitrary process.

This talk is based on Refs. [1, 2].

Fourth Annual Large Hadron Collider Physics

13-18 June 2016

Lund, Sweden

* Speaker.

${ }^{\dagger}$ I would like to thank S. Frixione, A. Papaefstathiou, S. Prestel and P. Torrielli for collaboration on Ref. [1] and K. Hamilton for collaboration on Ref. [2]. 


\begin{tabular}{c|lcc|c|l}
\hline & $\mu_{Q}=15 \mathrm{GeV}$ & $\mu_{Q}=25 \mathrm{GeV}$ & $\mu_{Q}=45 \mathrm{GeV}$ & inclusive & \\
\hline \hline \multirow{2}{*}{$Z+$ jets } & $2.055(-0.9 \%)$ & 2.074 & $2.085(+0.5 \%)$ & $2.012(-3.0 \%)$ & $\mathrm{HW}++$ \\
& $2.168(+0.8 \%)$ & 2.150 & $2.117(-1.5 \%)$ & $2.011(-6.5 \%)$ & $\mathrm{PY} 8$ \\
\hline \multirow{2}{*}{$W+$ jets } & $20.60(-0.9 \%)$ & 20.78 & $20.87(+0.4 \%)$ & $19.96(-3.9 \%)$ & $\mathrm{HW}++$ \\
& $21.71(+1.0 \%)$ & 21.50 & $21.18(-1.5 \%)$ & $19.97(-7.1 \%)$ & $\mathrm{PY} 8$ \\
\hline
\end{tabular}

Table 1: Total rates (in nb) for the three different choices of the FxFx merging scale, as well as those for the inclusive (i.e. non-merged) samples, obtained with HERwIG++ (upper rows) and PYTHIA8 (lower rows). Relative differences w.r.t. the FxFx results obtained with the central merging scale are also reported in brackets.

\section{Introduction}

During the last 15 years, the accuracy of the predictions for fully exclusive observables has been greatly improved. While even leading order (LO) multi-jet merging $[3,4,5,6,7,8,9,10,11$, 12] was rarely used and needed at the Tevatron, due to the much more precise data and higher QCD activity at the LHC, for many analyses next-to-leading order (NLO) multi-jet merging [13, 14, 15, $16,17,18,19,20,21,22,23,24]$ has become the new standard. These improved predictions are much more accurate and precise than their predecessors, allowing for a much higher scrutiny of the experimental data.

In this talk, two recent developments will be discussed in more detail: the data comparison for FxFx predictions for NLO vector boson plus multiple jets production and Minlo results for Higgs boson production with up to two jets at NLO accuracy.

\section{FxFx predictions for vector boson plus multi jets}

The FxFx merging method $[19,25]$ is a way for combining NLO predictions matched to the parton shower for various jet multiplicities. That is, it allows one to combine NLO matrix elements for $S+0 j, 1 j, 2 j, \ldots$ consistently over the whole phase-space. Here, $S$ is a system of particles that does not contain light partons (or $\mathrm{b}$ quarks). In the original publication, predictions for vector bosons, Higgs bosons and top pairs in association with up to two jets at NLO accuracy have been considered, matched to the HERwig6 parton shower. A carefull assessment of the predictions in comparison to data is still missing.

Following Ref. [1], we will present a comparison to $\mathrm{LHC} 7 \mathrm{TeV}$ vector boson plus jets data [26, 27, 28], as well as the validation of the extension of the method to more modern parton showers, i.e. Pythia8 [29] and Herwig++ [30]. The FxFx merged results include NLO matrix elements up to two jets at NLO accuracy (and therefore the third jet at LO accuracy). These results are also compared with the inclusive predictions, generated from the default matching of a vector boson with the parton shower at NLO accuracy, i.e. without the FxFx merging of higher jet multiplicities.

We start by considering the total cross sections, tabulated in Tab. 1. This table gives the total cross sections for $W$ and $Z$-boson production for the inclusive predictions and the FxFx merged results. For the latter, three different merging scales are used, $\mu_{Q}=15,25$ and $35 \mathrm{GeV}$. 
By construction, the inclusive cross sections are independent from the parton shower and are, in fact, equal to the fixed order total cross sections. The merged predictions are 3-4\% larger when matched to HeRwig++ and 6-7\% larger when matched to Pythia8. This is to be expected: the FxFx method is not a unitary approach and the inclusion of the (partial) higher order terms coming from the higher multiplicities indeed changes the inclusive cross section. The merging scale dependence is small: of the order of $1 \%$ for the total cross section and it is slightly smaller for HERWIG++ than for PYTHIA8.

We will now turn our attention to differential distributions. All the plots have the same layout: the green band (labeled 'Var' in the plots) is obtained by taking the envelop obtained from FxFx merging calculations with the $3 \times 3 \times 3$ scale variations (for the renormalisation, factorisation and merging scales, respectively). The red curve (labeled 'Inc.' in the plots) is the central value for the inclusive (i.e. non-merged) results. In the first inset the ratio w.r.t. the data is taken, where the yellow band shows the uncertainty on the data. The lowest inset displays the uncertainties coming from the 3 merging scales seperately as a ratio w.r.t. the central scale choice. The data are signified as black dots, and the triangles with the vertical green bars in the first inset are the central value and statistical uncertainty of the FxFx merged predictions.

We start by comparing $Z+$ jets $7 \mathrm{TeV}$ data from ATLAS [26] to our predictions in fig. 1 . In the left hand plots of this figure the data is compared to predictions made with the HERWIG++ parton shower; on the right with PYTHIA8. The upper plots contain the results for the exclusive jet multiplicity, the middle plots the transverse momentum of the hardest jet and the lowest plot the transverse momentum of the thrid hardest jet. Overall, the agreement between the FxFx merged results with the data is satisfactory, while the inclusive predictions undershoot the data considerable for the high jet multiplicities and/or large transverse momenta. More specifically, for the exclusive jet multiplicities, only the first three bins are formally NLO accurate, while the $N_{\text {jet }}=3$ bin is LO accurate for the FxFx merged results. For higher multiplicities the predictions are based purely on the parton shower. Indeed, the first 4 bins have a rather good agreement with data, which also means that the HeRwig++ and Pythia8 predictions lie on top of each other. For the higher jet multiplicities, HERWIG++ and PYTHIA8 start to differ and also the agreement with data is worse. For the transverse momentum of the hardest jet, there are some differences between the HERWIG++ and Pythia8 predictions, which shows that the effects of the parton shower cannot be neglected for this observable. For this observable, FxFx merging with PythiA8 is slighty preferred over the results matched with HERWIG++. A similar pattern can be seen for the transverse momentum of the third hardest jet, although the larger theory uncertainties (because this jet is only described at LO accuracy) show still an agreement with the data, even though the central value is slightly below for HERWIG++. For a larger selection of comparisons to $7 \mathrm{TeV}$ ATLAS Z-boson data, see Ref. [1].

In the $7 \mathrm{TeV}$ CMS results for Z-boson production in association with at least one hard jet [27], it was shown that there is a large difference between the data and the LO multi-jet predictions for the rapidity difference of the $Z$ boson and the hardest jet. A comparison with fixed order NLO $Z+$ jet showed agreement. Indeed, when using the FxFx multi-jet merging prediction, both for HeRwig++ and Pythia8 excellent agreement between the data and the prediction is found, see fig. 2. This is not the case for the inclusive (non-merged) results: there is a rather large difference between the predictions matching to HeRWIG++ and PythiA8 confirming that there is a large dependence on the parton shower when no higher multiplicity matrix elements are included in the 

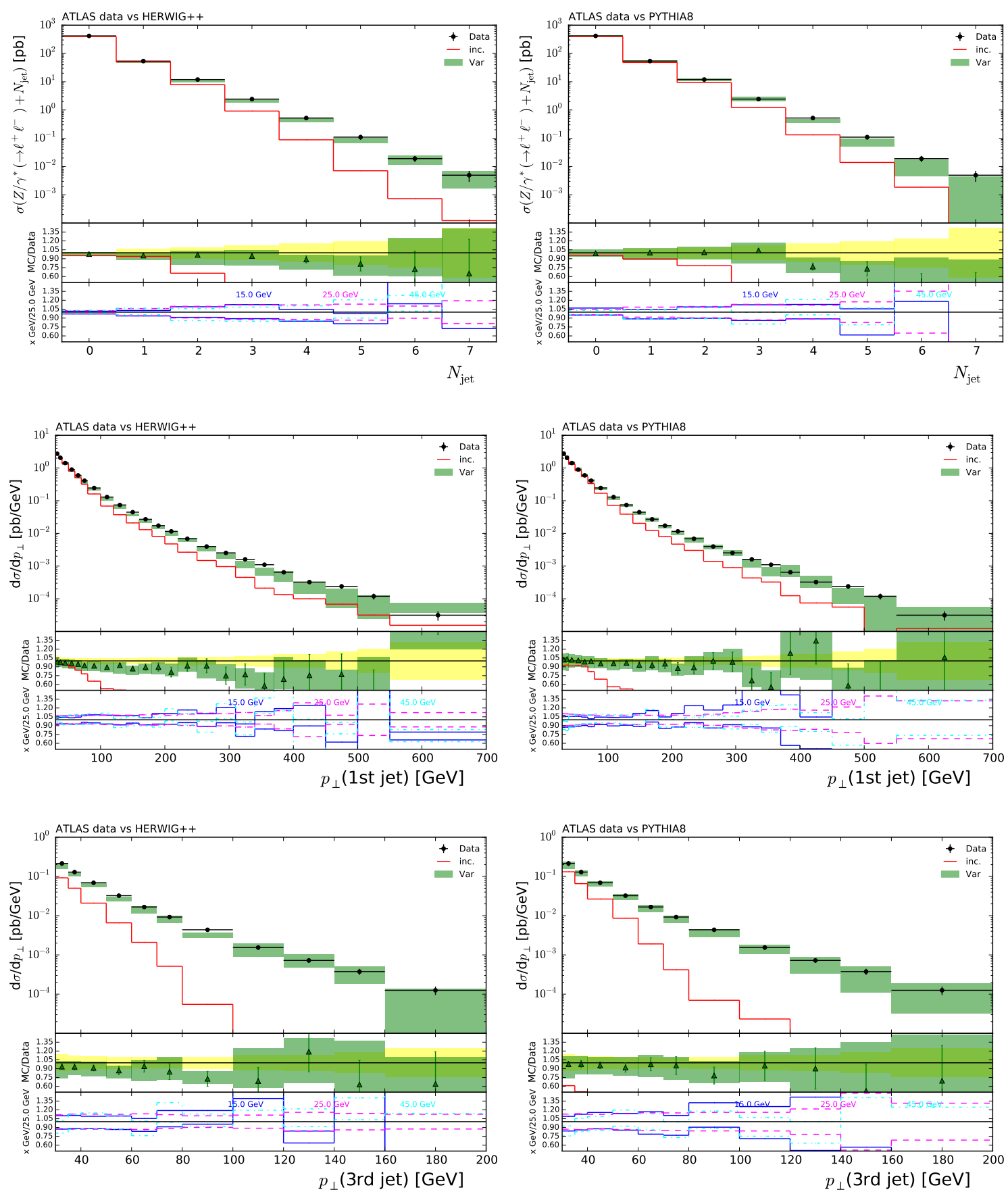

Figure 1: Exclusive jet multiplicity, transverse momentum of the $1^{\text {st }}$ jet and transverse momentum of the $3^{\text {rd }}$ jet. Data from ref. [26], compared to HERWIG++ (left panels) and PYTHIA8 (right panels) predictions. The FxFx uncertainty envelope ('Var') and the fully-inclusive central result ('inc') are shown as green bands and red histograms respectively.

results. For a larger selection of comparisons to $7 \mathrm{TeV}$ CMS Z-boson data, see Ref. [1].

Finally, we compare $7 \mathrm{TeV}$ ATLAS $W$-boson data [28] with the FxFx merged predictions. 

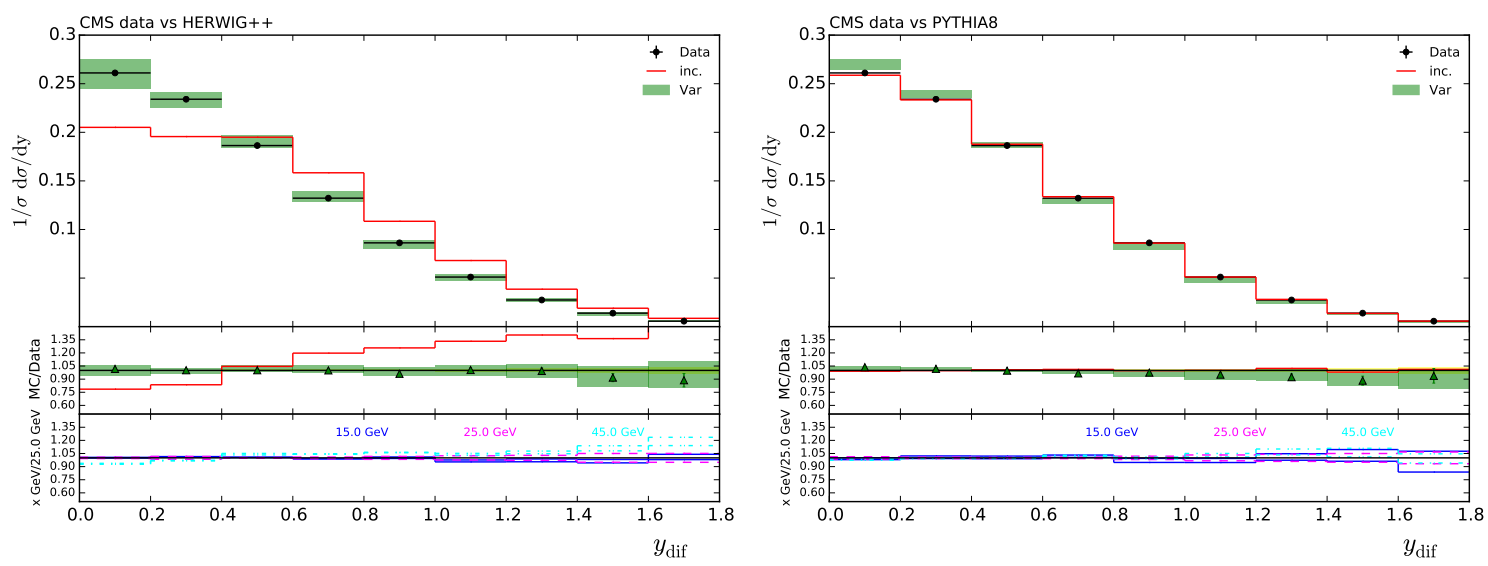

Figure 2: The difference of the rapidities of the $Z$ and the $1^{\text {st }}$ jet. Data from ref. [27], compared to HerWIG++ (left panel) and PYTHIA8 (right panel) predictions.

Similarly as for $Z$-boson production, agreement between predictions and data is satisfactory, except in cases where the shower plays a significant role. In the latter cases, also the differences between Herwig++ and Pythia8 are significant. In fig. 3 we show an example of the former, the azimuthal distance between the two hardest jets, where the agreement between theory and data is excellent, and also the invariant mass of the two hardest jets, where there is significant deviation between the data and the HERWIG++ predictions. Here, PYTHIA 8 does predict the data correctly. For a larger selection of comparisons to $7 \mathrm{TeV}$ ATLAS $W$-boson data as well as $7 \mathrm{TeV}$ CMS data [31], see Ref. [1].

\section{MiNLO for Higgs plus up to two jets}

The MINLO method [23] allows one to render an NLO calculation for $B+1$ jet calculation also NLO accurate for $B$ observables that are completely inclusive over the extra jets (with $B$ a massive colourless system of particles). This is quite a remarkable result, as fixed order results for $B+$ jet are singular for these observables. The idea behind the MINLO approach is to damp the singularity by applying a Sudakov factor and by including exactly the right terms inside this form factor, one can explicitly show that by expanding the full result, no terms that hamper the NLO accuracy of the predictions for inclusive $B$ production are left. Moreover, the form factor does not deteriorate the accuracy of the $B+1$ jet calculation. Even though higher order terms are needed in the Sudakov form factor, these terms do not -in general-improve the formal accuracy of the resummation.

In the original approach, the precise form of the Sudakov form factor was derived analytically. This allowed for predictions for $W / Z / H / H W$ production [23, 32], and, very recently, also $W W$ production [33]. In all these approaches it was possible to only cover up to one extra jet, i.e. starting from the NLO $B+1$ jet predictions also the $B$ inclusive observables become NLO accurate. In the following we will show some results on an alternative method ("extended MinLO") [2], which uses unitarity to enforce NLO $B+\mathrm{n}$-jet predictions to be also NLO accurate for $B+(\mathrm{n}-1)$ jet observables. In particular, results will be shown for Higgs production in gluon fusion, in which we start from an NLO calculation for $H+2$ jets and apply the extended MINLO method to make them 

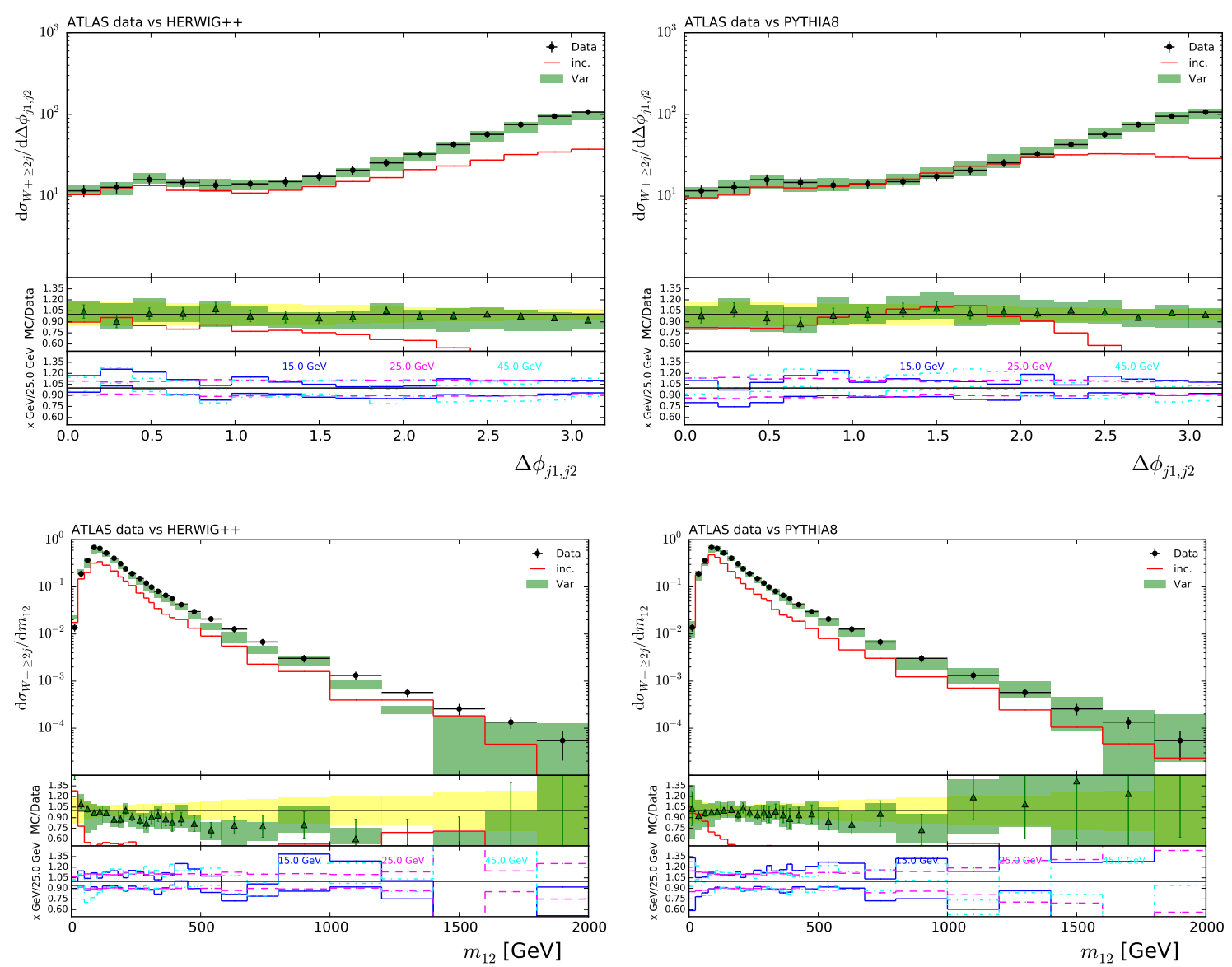

Figure 3: The azimuthal distance between and the invariant mass of the two hardest jets. Data from ref. [28], compared to HERWIG++ (left panels) and PYTHIA8 (right panels) predictions.

also accurate in $H+1$ jet predictions. The latter are already part of an NNLO+PS calculation for Higgs production [34]. In short, this allows one to have NLO accuracy for $H+2$ jets and $H+1$ jet and NNLO accuracy for inclusive $H$ observables without the introduction of a merging scale. The results shown here are just a proof-of-concept and not yet complete enough for data comparisons: at high transverse momenta (or other large scales) the effective theory used, i.e. integrating out the top quark in the Higgs to gluons coupling, breaks down and improvements are needed [35, 36].

The most interesting result from this exercise is the plot of fig. 4. In this plot, the transverse momentum of the Higgs boson is plotted, requiring two additional jets in the event, vetoing events with more or fewer jets. In the left panel, three predictions are shown: in red are the new extended MINLO results, in green the central value of the NNLOPS calculation for inclusive $H$ production and in blue the predictions for $H+2$ jets are shown. The latter contains Sudakov resummation, but is formally not NLO accurate for lower multiplicity observables. In the three panels on the right, the ratio w.r.t. the three central values is taken, respectively. The coloured band is the uncertainty coming from scale variations in the respective results. As expected, when the transverse momentum of the Higgs boson is small (compared to the typical transverse momenta of the jets) the extended MiNLO results agree with the calculation for $H+2$ jets. However, when the transverse momentum 
of the Higgs gets larger, the events are dominated by the Higgs recoiling against a hard jet, that either splits into two, or radiates a softer secondary jet. The latter approach is better described by the NLO predictions for $H+1$ jet: indeed, the extended MINLO results agree with the NNLOPS results for inclusive $H$ production. A much larger set of results and comparisons can be found in Ref. [2].
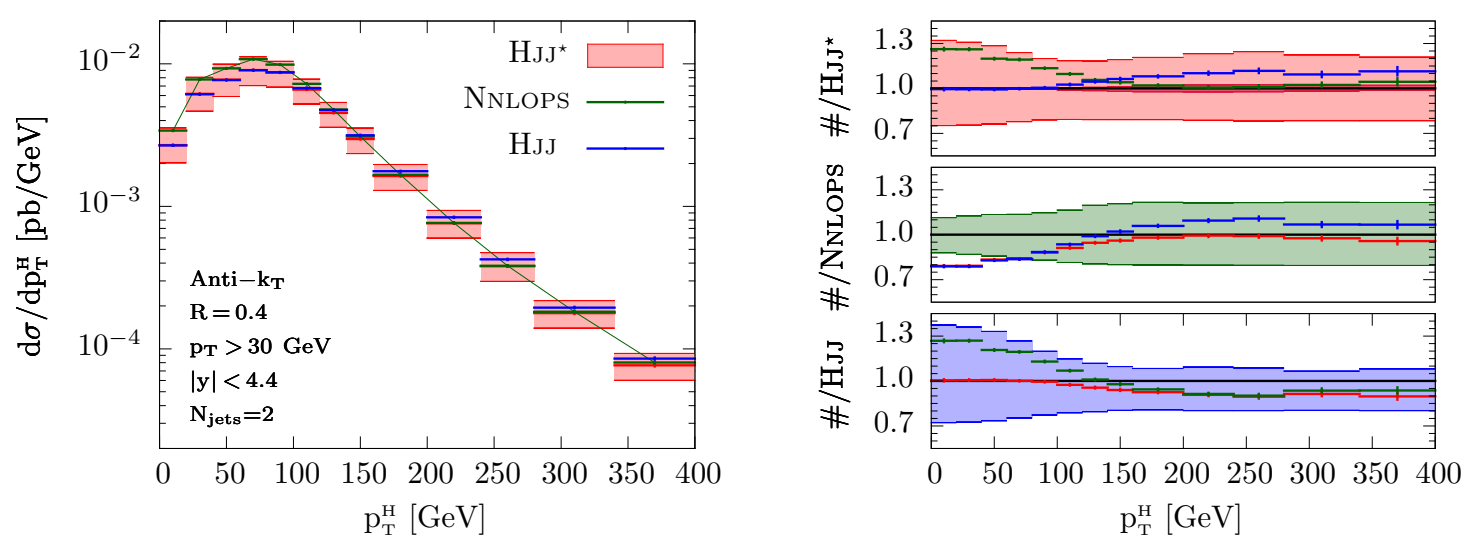

Figure 4: Transverse momentum distribution of the Higgs boson in 2-jet events. Jets are here constructed according to the anti- $k_{t}$ clustering algorithm, for a radius parameter $R=0.4$. Jets are required to have transverse momentum $p_{\mathrm{T}} \geq 30 \mathrm{GeV}$ and rapidity $|\mathrm{y}| \leq 4.4$.

\section{Conclusions}

During the last decade(s) fully exclusive predictions for event rates and differential distributions have improved enormously. Both in precision as well as accuracy. In this talk, I have presented two examples of recent progress.

The first example is a data comparison between FxFx merged predictions and $7 \mathrm{TeV}$ LHC data for $W$ and $Z$ production in association with multiple jets. Even though a small, representative selection of the full results is shown it can be concluded that overall the agreement between the predictions and the data is satisfactory. For the observables for which deviations with the data are found, also large differences between matching to HeRWIG++ and PYTHIA8 are found. In a way this is good news, as it allows one to consider predictions for which HERWIG++ and PYTHIA8 agree very robust.

The second example are currently the most accurate predictions for Higgs boson production in gluon fusion, merging NLO $H+2$ jets with NNLO $H$ results, without the introduction of a merging scale. Using unitarity in a numerical way, no new analytic calculations are needed to extend this approach to other processes.

It took about ten years in going from the first NLO results matched to a parton shower [37, 38, 39], to fully fledged automation [40, 25, 41, 42]. With the first results for next-to-next-to-leading order predictions matched to parton showers coming available today [34, 43, 44, 45, 46, 32], it will be interesting to see how far we can push the field in the coming decade. 


\section{Acknowledgements}

The speaker is supported by the Alexander von Humboldt Foundation, in the framework of the Sofja Kovalevskaja Award Project "Event Simulation for the Large Hadron Collider at High Precision".

\section{References}

[1] R. Frederix, S. Frixione, A. Papaefstathiou, S. Prestel and P. Torrielli, JHEP 1602 (2016) 131 doi:10.1007/JHEP02(2016)131 [arXiv:1511.00847 [hep-ph]].

[2] R. Frederix and K. Hamilton, JHEP 1605 (2016) 042 doi:10.1007/JHEP05(2016)042 [arXiv:1512.02663 [hep-ph]].

[3] S. Catani, F. Krauss, R. Kuhn and B. R. Webber, JHEP 0111 (2001) 063 doi:10.1088/1126-6708/2001/11/063 [hep-ph/0109231].

[4] L. Lonnblad, JHEP 0205 (2002) 046 doi:10.1088/1126-6708/2002/05/046 [hep-ph/0112284].

[5] F. Krauss, JHEP 0208 (2002) 015 doi:10.1088/1126-6708/2002/08/015 [hep-ph/0205283].

[6] S. Mrenna and P. Richardson, JHEP 0405 (2004) 040 doi:10.1088/1126-6708/2004/05/040 [hep-ph/0312274].

[7] N. Lavesson and L. Lonnblad, JHEP 0507 (2005) 054 doi:10.1088/1126-6708/2005/07/054 [hep-ph/0503293].

[8] J. Alwall et al., Eur. Phys. J. C 53 (2008) 473 doi:10.1140/epjc/s10052-007-0490-5 [arXiv:0706.2569 [hep-ph]].

[9] S. Hoeche, F. Krauss, S. Schumann and F. Siegert, JHEP 0905 (2009) 053 doi:10.1088/1126-6708/2009/05/053 [arXiv:0903.1219 [hep-ph]].

[10] K. Hamilton, P. Richardson and J. Tully, JHEP 0911 (2009) 038 doi:10.1088/1126-6708/2009/11/038 [arXiv:0905.3072 [hep-ph]].

[11] L. Lonnblad and S. Prestel, JHEP 1203 (2012) 019 doi:10.1007/JHEP03(2012)019 [arXiv:1109.4829 [hep-ph]].

[12] L. Lonnblad and S. Prestel, JHEP 1302 (2013) 094 doi:10.1007/JHEP02(2013)094 [arXiv:1211.4827 [hep-ph]].

[13] N. Lavesson and L. Lonnblad, JHEP 0812 (2008) 070 doi:10.1088/1126-6708/2008/12/070 [arXiv:0811.2912 [hep-ph]].

[14] K. Hamilton and P. Nason, JHEP 1006 (2010) 039 doi:10.1007/JHEP06(2010)039 [arXiv:1004.1764 [hep-ph]].

[15] S. Hoche, F. Krauss, M. Schonherr and F. Siegert, JHEP 1108 (2011) 123 doi:10.1007/JHEP08(2011)123 [arXiv:1009.1127 [hep-ph]].

[16] W. T. Giele, D. A. Kosower and P. Z. Skands, Phys. Rev. D 84 (2011) 054003 doi:10.1103/PhysRevD.84.054003 [arXiv:1102.2126 [hep-ph]].

[17] S. Alioli, K. Hamilton and E. Re, JHEP 1109 (2011) 104 doi:10.1007/JHEP09(2011)104 [arXiv:1108.0909 [hep-ph]]. 
[18] S. Hoeche, F. Krauss, M. Schonherr and F. Siegert, JHEP 1304 (2013) 027 doi:10.1007/JHEP04(2013)027 [arXiv:1207.5030 [hep-ph]].

[19] R. Frederix and S. Frixione, JHEP 1212 (2012) 061 doi:10.1007/JHEP12(2012)061 [arXiv:1209.6215 [hep-ph]].

[20] S. Plätzer, JHEP 1308 (2013) 114 doi:10.1007/JHEP08(2013)114 [arXiv:1211.5467 [hep-ph]].

[21] S. Alioli, C. W. Bauer, C. J. Berggren, A. Hornig, F. J. Tackmann, C. K. Vermilion, J. R. Walsh and S. Zuberi, JHEP 1309 (2013) 120 doi:10.1007/JHEP09(2013)120 [arXiv:1211.7049 [hep-ph]].

[22] L. Lönnblad and S. Prestel, JHEP 1303 (2013) 166 doi:10.1007/JHEP03(2013)166 [arXiv:1211.7278 [hep-ph]].

[23] K. Hamilton, P. Nason, C. Oleari and G. Zanderighi, JHEP 1305 (2013) 082 doi:10.1007/JHEP05(2013)082 [arXiv:1212.4504 [hep-ph]].

[24] S. Alioli, C. W. Bauer, C. Berggren, F. J. Tackmann, J. R. Walsh and S. Zuberi, JHEP 1406 (2014) 089 doi:10.1007/JHEP06(2014)089 [arXiv:1311.0286 [hep-ph]].

[25] J. Alwall et al., JHEP 1407 (2014) 079 doi:10.1007/JHEP07(2014)079 [arXiv:1405.0301 [hep-ph]].

[26] G. Aad et al. [ATLAS Collaboration], JHEP 1307 (2013) 032 doi:10.1007/JHEP07(2013)032 [arXiv:1304.7098 [hep-ex]].

[27] S. Chatrchyan et al. [CMS Collaboration], Phys. Rev. D 88 (2013) no.11, 112009 doi:10.1103/PhysRevD.88.112009 [arXiv:1310.3082 [hep-ex]].

[28] G. Aad et al. [ATLAS Collaboration], Eur. Phys. J. C 75 (2015) no.2, 82 doi:10.1140/epjc/s10052-015-3262-7 [arXiv:1409.8639 [hep-ex]].

[29] T. Sjöstrand et al., Comput. Phys. Commun. 191 (2015) 159 doi:10.1016/j.cpc.2015.01.024 [arXiv:1410.3012 [hep-ph]].

[30] M. Bahr et al., Eur. Phys. J. C 58 (2008) 639 doi:10.1140/epjc/s10052-008-0798-9 [arXiv:0803.0883 [hep-ph]].

[31] V. Khachatryan et al. [CMS Collaboration], Phys. Lett. B 741 (2015) 12 doi:10.1016/j.physletb.2014.12.003 [arXiv:1406.7533 [hep-ex]].

[32] W. Astill, W. Bizon, E. Re and G. Zanderighi, doi:10.1007/JHEP06(2016)154 arXiv:1603.01620 [hep-ph].

[33] K. Hamilton, T. Melia, P. F. Monni, E. Re and G. Zanderighi, arXiv:1606.07062 [hep-ph].

[34] K. Hamilton, P. Nason, E. Re and G. Zanderighi, JHEP 1310 (2013) 222 doi:10.1007/JHEP10(2013)222 [arXiv:1309.0017 [hep-ph]].

[35] K. Hamilton, P. Nason and G. Zanderighi, JHEP 1505 (2015) 140 doi:10.1007/JHEP05(2015)140 [arXiv:1501.04637 [hep-ph]].

[36] R. Frederix, S. Frixione, E. Vryonidou and M. Wiesemann, JHEP 1608 (2016) 006 doi:10.1007/JHEP08(2016)006 [arXiv:1604.03017 [hep-ph]].

[37] S. Frixione and B. R. Webber, JHEP 0206 (2002) 029 doi:10.1088/1126-6708/2002/06/029 [hep-ph/0204244].

[38] S. Frixione, P. Nason and B. R. Webber, JHEP 0308 (2003) 007 doi:10.1088/1126-6708/2003/08/007 [hep-ph/0305252]. 
[39] P. Nason, JHEP 0411 (2004) 040 doi:10.1088/1126-6708/2004/11/040 [hep-ph/0409146].

[40] S. Alioli, P. Nason, C. Oleari and E. Re, JHEP 1006 (2010) 043 doi:10.1007/JHEP06(2010)043 [arXiv:1002.2581 [hep-ph]].

[41] S. Hoche, F. Krauss, M. Schonherr and F. Siegert, JHEP 1104 (2011) 024 doi:10.1007/JHEP04(2011)024 [arXiv:1008.5399 [hep-ph]].

[42] S. Hoeche, F. Krauss, M. Schonherr and F. Siegert, JHEP 1209 (2012) 049 doi:10.1007/JHEP09(2012)049 [arXiv:1111.1220 [hep-ph]].

[43] S. Höche, Y. Li and S. Prestel, Phys. Rev. D 91 (2015) no.7, 074015 doi:10.1103/PhysRevD.91.074015 [arXiv:1405.3607 [hep-ph]].

[44] S. Höche, Y. Li and S. Prestel, Phys. Rev. D 90 (2014) no.5, 054011 doi:10.1103/PhysRevD.90.054011 [arXiv:1407.3773 [hep-ph]].

[45] A. Karlberg, E. Re and G. Zanderighi, JHEP 1409 (2014) 134 doi:10.1007/JHEP09(2014)134 [arXiv:1407.2940 [hep-ph]].

[46] S. Alioli, C. W. Bauer, C. Berggren, F. J. Tackmann and J. R. Walsh, Phys. Rev. D 92 (2015) no.9, 094020 doi:10.1103/PhysRevD.92.094020 [arXiv:1508.01475 [hep-ph]]. 